\title{
DEFINICIÓN DE LA ÉPOCA DE INCENDIOS FORESTALES EN UN CONTEXTO MULTIVARIADO
}

Marcos Pedro Ramos Rodríguez ${ }^{(1)}$

Yudisnelvis González Menzonet ${ }^{(2)}$

\begin{abstract}
RESUMEN
En este trabajo se presenta un acercamiento a la definición de la época de incendios forestales en un contexto multivariado como contribución a la planificación del manejo de los mismos. El método se basa en seleccionar las variables que expresen el comportamiento histórico de los incendios cada mes durante un periodo de años determinado. Entre estas variables se encuentran la densidad de incendios y la densidad de afectaciones, la media y la mediana del área quemada por incendio, el número de grandes incendios y la máxima área quemada por un incendio. Usando sistemas estadísticos pueden agruparse los meses con técnicas de análisis de cluster y la época de incendio puede ser definida. El método se aplicó en las provincias Santiago de Cuba y Pinar del Río, ubicadas en las regiones oriental y occidental de Cuba respectivamente. Se utilizaron estadísticas de incendios del periodo 1997 - 2002.

Palabras Claves: Comportamiento histórico, manejo de incendios, análisis de cluster, estadísticas de incendios.
\end{abstract}

\section{DEFINITION OF THE FIRE SEASON IN A MULTIVARIATE CONTEXT}

\begin{abstract}
In this work an approach to the definition of the fire season in a multivariate context is presented. It is a contribution to the forest fire management. The method is based on selecting the variables that express the fire history every month during a certain period of years. Among these variables there are the density of fires and the density of affectations, the mean and median of the area burned by fire, the number of big fires and the maximum area burned by a fire. To group the months is used the cluster analysis technical and it allows to define the fire season. The method was applied in the provinces of Santiago de Cuba and Pinar del Río, located in the regions oriental and western of Cuba respectively. Fire statistics from the period 1997/2002 were used.

Keywords: Fire history, fire management, cluster analysis, fire statistics.
\end{abstract}

\section{INTRODUCCIÓN}

Cada año los incendios forestales se presentan con mayor frecuencia en determinados meses del año. Este periodo es conocido como época de incendios, época de ocurrencias ó época crítica. Según Soares (1985) las épocas de mayor ocurrencias de incendios durante el año pueden variar bastante entre las regiones, especialmente en países de grandes dimensiones territoriales. Esas variaciones son causadas principalmente por el clima, aunque pueden ser influenciadas por los tipos de vegetación.

Definir en cada territorio la época de

incendios es de gran importancia para la planificación del manejo del fuego en los mismos. Para lograr esto, tradicionalmente se utilizan las estadísticas de los incendios durante un periodo de años y con estas se elabora una tabla en la cual es posible observar los porcentajes de los incendios ocurridos y de las hectáreas por ellos afectadas cada mes del año. Esto, además, se plotea en un gráfico. De esta forma es posible definir los meses durante los cuáles, a través del periodo de años, han ocurrido la mayor cantidad de incendios y/o las mayores afectaciones. Se ha observado que

\footnotetext{
${ }^{1}$ Profesor, Ingeniero Forestal. Universidad de Pinar del Río, Cuba. Apartado Postal 268, Pinar del Río 1, Código: 20100, Cuba. E-mail: cmramos@af.upr.edu.cu

${ }^{2}$ Estudiante de Quinto Año de Ingeniería Forestal. Universidad de Pinar del Río, Cuba. Calle Martí No. 270, Esquina 27 de Noviembre. Pinar del Río 1, Código: 20100, Cuba.
} 
en algunos territorios los meses de mayores valores para ambas variables no coinciden. Surgen entonces dificultades para definir el periodo que debe adoptarse como el de mayores probabilidades de surgimiento $\mathrm{y}$ propagación de los incendios forestales (época de incendios). Además, estas dos variables son buenos indicadores del comportamiento histórico de los incendios, pero también existen otras menos usadas que pueden ayudar a expresar dicho comportamiento con mayor precisión.

Teniendo como antecedentes el trabajo "Land zoning based on fire history" (Bobio, et al., 1997) se ha considerado que la problemática descrita anteriormente puede resolverse utilizando el análisis multivariado conocido como análisis de conglomerados (Cluster Analysis). Este análisis consiste según González (1997) en el empleo de algoritmos que tienen por objeto la búsqueda de grupos similares de variables o de observaciones, por tanto, a partir de una serie de variables de las que se posee un conjunto de observaciones, el análisis de conglomerado agrupa (tanto variables como observaciones) en grupos los más homogéneos posible, por lo que las características de todos los componentes de un grupo serán similares.

En este trabajo se presenta un acercamiento a la definición de la época de incendios forestales en un contexto multivariado como contribución a la planificación del manejo de los mismos en las provincias Santiago de Cuba y Pinar del Río, ubicadas en las regiones oriental y occidental de Cuba respectivamente.

\section{MATERIALES Y MÉTODOS}

La provincia de Santiago de Cuba tiene una superficie cubierta de bosques de 166 758,10 hectáreas y Pinar del Río 376 112,31 hectáreas. En la primera provincia predominan las latifolias y en la segunda las coníferas. En ambos territorios los incendios se distribuyen durante el año similar a como ocurre en el resto del país en el cual se ha definido la época de incendios en marzo y abril. La topografía en ambas regiones es variada, no obstante, la vegetación forestal se localiza fundamentalmente en zonas montañosas. En Pinar del Río el problema de los incendios es considerado más serio que en Santiago de Cuba. El número de incendios es aproximadamente 16,31 veces superior y el área quemada 9,22 veces más extensa.

Para el estudio se consideró en ambas provincias un periodo de 6 años (1997 - 2002). Los datos fueron obtenidos en las Direcciones del Cuerpo de Guardabosques de ambas provincias. Su procesamiento se realizó con el Sistema para el Manejo de Bases de Datos sobre Incendios Forestales (SIMBDIF V. 1.2) (Ramos, 2000). El procesamiento estadístico se realizó con los sistemas NTSYS-pc V. 1.80 y SPSS V. 10.

El algoritmo del trabajo consideró la selección de las variables, la elección de la medida de similaridad, la elección del método de agregación (agrupamiento), la validación estadística del agrupamiento y la definición de los grupos.

\section{Selección de las variables}

Las variables seleccionadas expresan en su conjunto el comportamiento histórico de los incendios forestales cada mes del año durante el periodo del estudio. De acuerdo con esto, se definieron 10 variables que pueden dividirse en dos grupos: uno relacionado con la ocurrencia de los incendios y otro con la propagación del fuego. Estas variables son las siguientes:

- Densidad de incendios (DI): Variable definida en la Norma Ramal de la Agricultura 555 (MINAG, 1982). Expresa el número medio de los incendios ocurridos en el periodo analizado por 1000 ha cubiertas de bosques, cada uno de los meses del año.

- Densidad de afectaciones (DA): Variable definida también en la Norma Ramal de la Agricultura 555 (MINAG, 1982). Es la media aritmética del área quemada en el periodo analizado por 1000 ha de superficie cubierta de bosque, cada uno de los meses del año.

- Media del área quemada por incendio (AQI): Es la media de las hectáreas quemadas por incendio en el periodo que se analiza, cada uno de los meses del año.

- Mediana del área quemada por incendio (MAQI): Esta variable define el tamaño del incendio típico ocurrido cada mes, no estando afectado por los valores extremos de la distribución, como ocurre en el caso de la media del área quemada por incendio. 
- Máxima área quemada por un incendio (MxAQ): Se refiere al mayor incendio ocurrido cada mes del año durante el periodo analizado. Indica la máxima severidad que el fuego desarrolló cada mes.

- Densidad de grandes incendios (DGI): Es la media aritmética del área quemada por grandes incendios, en el periodo analizado por 1000 ha de superficie cubierta de bosque, cada uno de los meses del año. Se consideraron incendios grandes a aquellos que afectaron más de 40 ha, valor a partir del cual se agrupan a los incendios en las clases de tamaño IV y $\mathrm{V}$ según Ramsey e Higgins (1981) citados por Soares (1992).

- Porcentaje del número de años en los cuales, en cada mes, ocurrieron más de " $X$ " incendios (NAI): Para determinar el número " $\mathrm{X}$ " de incendios se hace una distribución de frecuencias para el número de incendios ocurridos cada mes en cada uno de los años del periodo y se desprecian las clases cuya frecuencia supere el $30 \%$ del total de incendios de la clase en que estos más se repiten. De acuerdo con las figuras 1 y 2 este número para Santiago de Cuba fue $2 \mathrm{y}$ para Pinar del Río, 10.

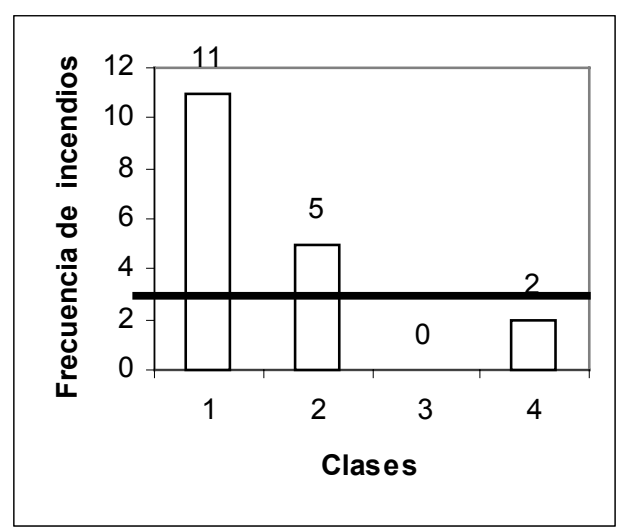

Figura 1: Distribución de frecuencia para el número de incendios cada mes en Santiago de Cuba Figure 1: Distribution of frequency for number of forest fire each month in Santiago de Cuba

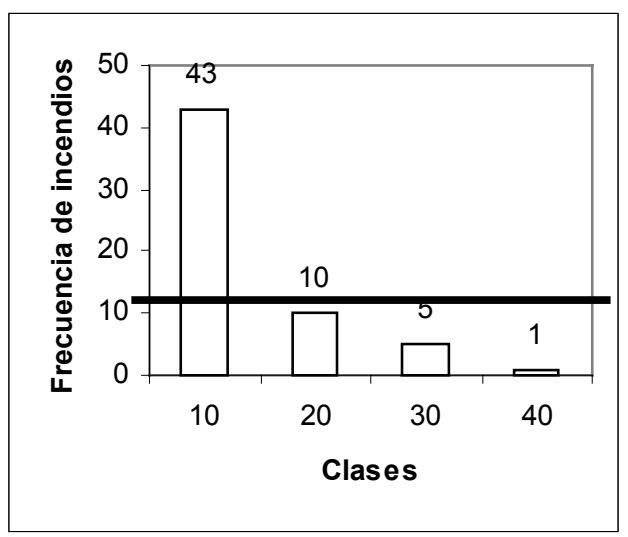

Figura 2: Distribución de frecuencia para el número de incendios cada mes en Pinar del Río

Figure 2: Distribution of frequency for number of forest fire each month in Pinar del Río

- Porcentaje del número de días de cada mes en los cuales ocurrieron incendios (NDI). De esta forma se tratan de diferenciar meses en los cuales han ocurrido incendios en un porcentaje alto de sus días de los que ha ocurrido lo contrario.

- Porcentaje de número de horas del día en las cuáles, en cada mes, ocurrieron más de " $X$ " incendios (NHI). El número "X" de incendios se establece haciendo una distribución de frecuencias para el número de incendios ocurridos cada hora del día en cada uno de los meses del año y se desprecian las clases cuya frecuencia supere el $30 \%$ del total de incendios de la clase en que estos más se repiten. De acuerdo con las figuras 3 y 4 este 
número para Santiago de Cuba fue $1 \mathrm{y}$ para Pinar del Río, 5.

\section{b) Elección de la medida de similaridad}

Medir la similitud o disimilitud entre los casos, entidades, objetos u OTUs (Operational Taxonomic Unit) a partir de las variables, atributos o características que los identifican es fundamental para el proceso de clasificación. Según Aldenderfer, et al. (1987), Sneath y Sokal (1973) subdividieron al coeficiente de similitud en cuatro grupos: coeficiente de correlación, medición de las distancias, coeficiente de asociación y medición probabilística de la similitud. Para los propósitos del trabajo se recomienda utilizar alguno de los métodos del segundo grupo, es decir, de la medición de distancias, siendo los más comunes la distancia Euclídea y la Manhattan.

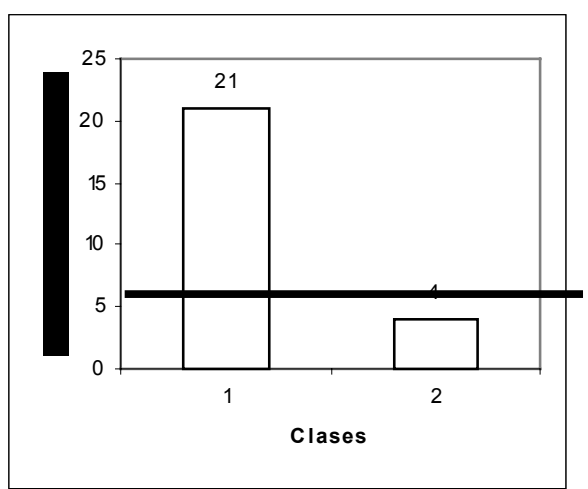

Figura 3: Distribución de frecuencia para el número de incendios por horas cada mes en Santiago de Cuba Figure 3: Distribution of frequency for number of forest fire for hours each month in Santiago de Cuba

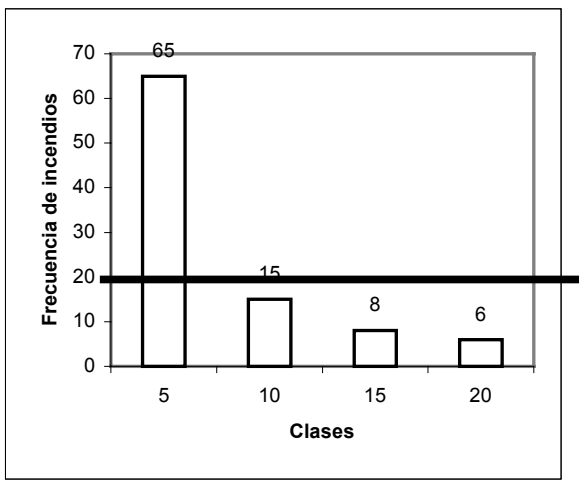

Figura 4. Distribución de frecuencia para el número de incendios por horas cada mes en Pinar del Río. Figure 4. Distribution of frequency for number of forest fire for hours each month in Pinar del Río.

\section{c) Método de agregación (agrupamiento)}

La primera razón para el uso del análisis de conglomerados es encontrar grupos de entidades similares en una muestra de datos (Aldenderfer, et al., 1987). Se utilizará en este trabajo el método de agrupamiento o clasificación jerárquica en el cual (Cuadras, 1991) los grupos se van fusionando progresivamente, mientras decrece la homogeneidad entre los grupos, cada vez más amplios, que se van formando. Según Aldenderfer, et al. (1987) en una revisión de todos los artículos publicados y que usaron el análisis de conglomerado durante 1973, Blashfield y Aldenderfer (1978) encontraron que dos tercios de esos artículos presentaba algún método de agrupamiento jerárquico. En este caso los métodos más comunes son el método del vecino más cercano o de ligamiento simple (Single Linkage), el del vecino más lejano o de ligamiento completo (Complete Linkage), el del ligamiento promedio no ponderado (Unweighted Pair - 
Group Average) (UPGA) y el de Ward o del error de la suma de cuadrados.

\section{d) Validación estadística del agrupamiento}

Se utilizó el coeficiente de correlación cofenética, introducido según Cuadras (1991) por Sokal y Rohlf en 1962 como medida del grado de buena clasificación. Este coeficiente es usado (Aldenderfer, et al., 1987) para determinar cómo el árbol o dendrograma resultado de un método jerárquico representa el modelo de similitud / disimilitud entre las entidades. Cuando el coeficiente es próximo a 1, existe una clara estructura entre los objetos. Valores bajos del coeficiente indican una distorsión notable entre las disimilitudes iniciales y las que resultan del dendrograma.

\section{e) Definición de los grupos}

Se utilizará el procedimiento heurístico, el cual según Aldenderfer, et al. (1987) ha sido por mucho tiempo el más comúnmente usado. Con este método el dendrograma es cortado a través de una inspección subjetiva a diferentes niveles. Es un procedimiento muy satisfactorio porque generalmente se basa en las necesidades y opiniones del investigador.

\section{RESULTADOS Y DISCUSIÓN}

Las figuras 5 y 6 muestran para cada una de las provincias objeto de análisis la distribución del porcentaje mensual de los incendios y las hectáreas de bosques por ellos afectadas en el periodo. De acuerdo con ellas, como se observa, no en todos los meses con mayor cantidad de incendios se produjeron también las mayores afectaciones. Se observa también que las épocas de incendios que se pudieran establecer de acuerdo con alguna de las dos variables no coincidirían. Esto indica la necesidad de hacer el análisis en un contexto multivariado.

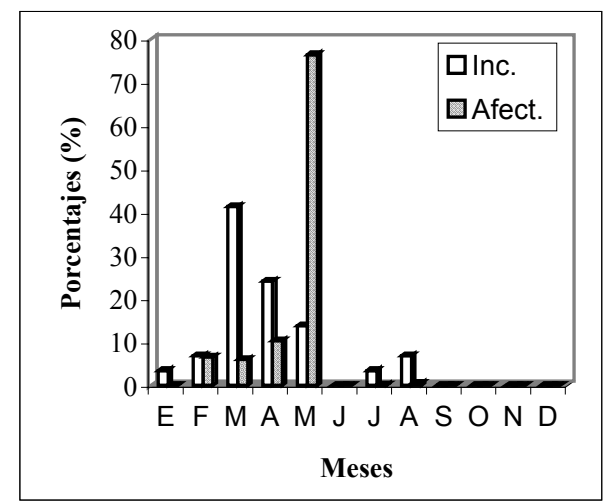

Figura 5: Porcentajes de incendios y sus áreas quemadas por mes en Santiago de Cuba 1997 - 2002

Figure 5: Percent of forest fire and burn area by month in Santiago de Cuba 1997 - 2002

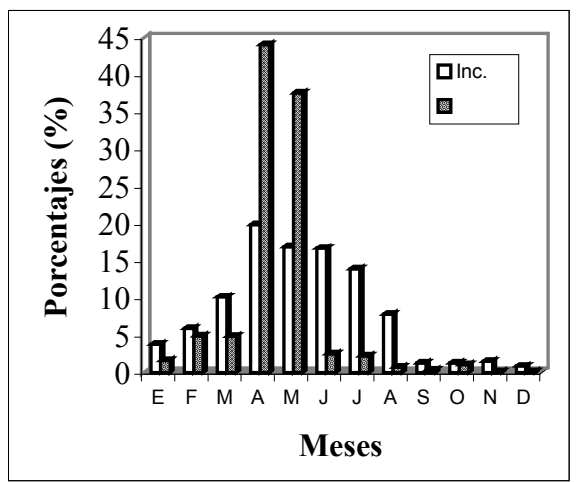

Figura 6: Porcentajes de incendios y sus áreas quemadas por mes en Pinar del Río 1997 - 2002

Figure 6: Percent of forest fire and burn area by month in Pinar del Rio 1997 - 2002

Para la obtención de los dendrogramas primero se estandarizaron los datos de la matriz original y después se fueron probando las medidas de distancias manhattan y euclidea con los métodos de agrupamiento del vecino más cercano (ligamiento simple), el del vecino 
más lejano (ligamiento completo) y el del ligamiento promedio no ponderado (UPGA). La combinación que mostró el mayor coeficiente de correlación cofenética en ambos casos fue la distancia euclidea con el método del ligamiento completo. Sus valores fueron de 0,98 para Santiago de Cuba y de 0,90 para Pinar del Río.
Los dendrogramas de las figuras 7 y 8 muestran los grupos de meses obtenidos de acuerdo a la similitud o disimilitud existente entre los mismos en Santiago de Cuba y Pinar del Río respectivamente. En ambos casos según va aumentando la distancia, se van uniendo grupos entre sí hasta formar un único grupo.

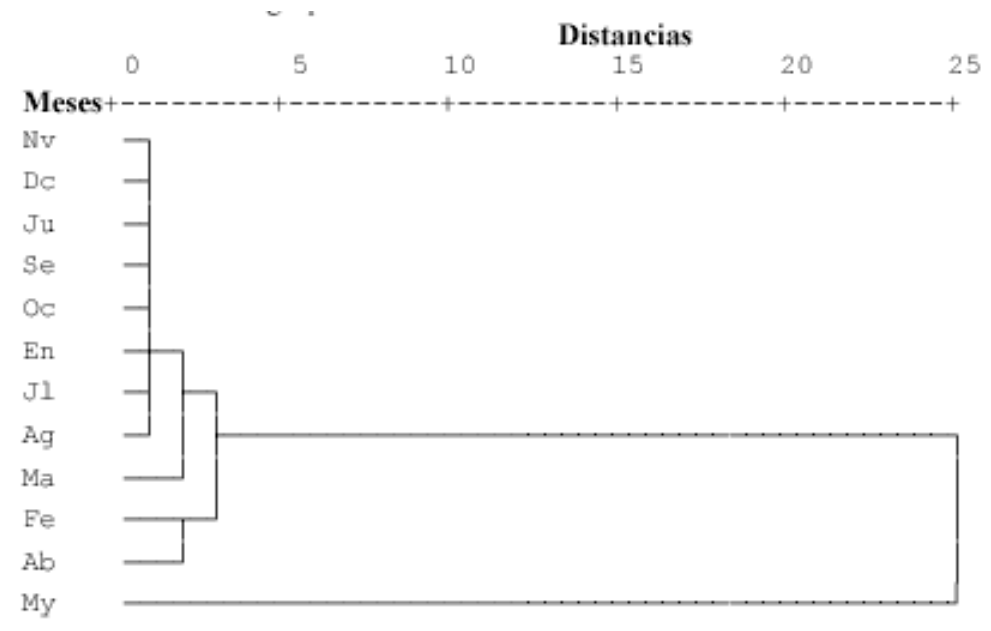

Figura 7: Dendrograma para Santiago de Cuba

Figure7: Dendrogram of Santiago de Cuba

Teniendo en cuenta los dendrogramas anteriores y observando las figuras 5 y 6 es posible definir para cada una de las provincia la época de incendios. En ambos casos la misma debe extenderse desde febrero a mayo. En el caso de Santiago de Cuba de acuerdo con la figura 5, febrero pudiera excluirse de la época de incendios. Sin embargo, en el dendrograma de la figura 7 se observa que febrero es similar a abril a una distancia bastante pequeña. No existen dudas entonces para incluir a febrero en la época de incendios. En el caso de Pinar del Río, si se observa la figura 6, la duda estaría en la inclusión o no de los meses junio, julio y agosto en la época de incendios. De acuerdo con el dendrograma de la figura 8 estos meses son similares a una distancia pequeña a meses tales como enero, octubre, noviembre y diciembre en los cuales tanto la ocurrencia como la propagación del fuego alcanzan bajos niveles por lo que no se incluirían en la época de incendios.

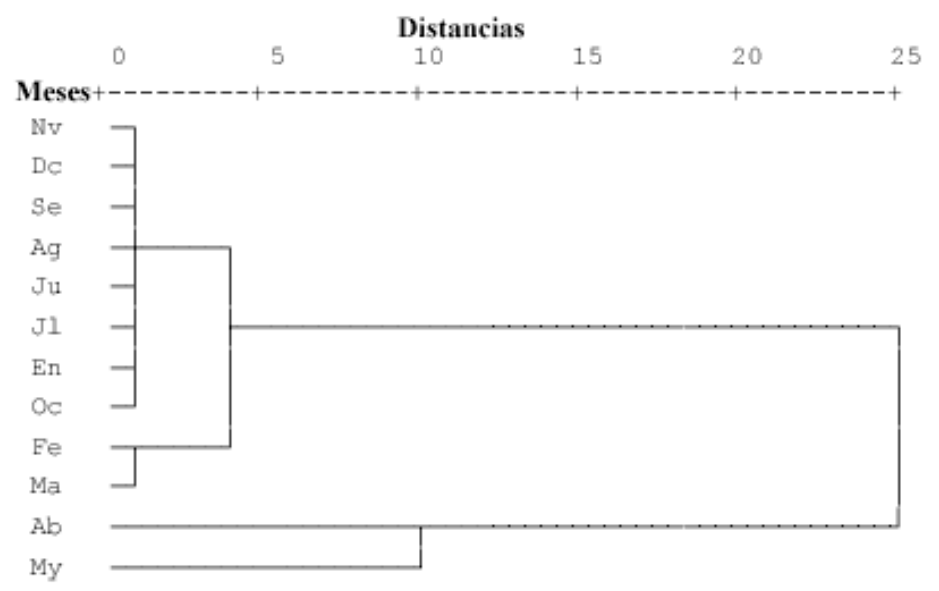

Figura 8: Dendrograma para Pinar del Río 
Figure 8: Dendrogram of Pinar del Río

\section{CONCLUSIONES}

A partir de bases de datos de incendios forestales pueden obtenerse variables que expresan el comportamiento histórico de los mismos. Estas variables al analizarse en un contexto multivariado, permiten definir con facilidad y con mayor fundamentación la época de incendios en un territorio determinado.

En este trabajo se ha definido la época de incendios para las provincias de Santiago de Cuba y Pinar del Río entre los meses de febrero a mayo.

\section{REFERÊNCIAS}

Aldenderfer, M. S. y R. K. Blashfield. 1987: Cluster Analysis. Cuarta Reimpresión. SAGE Publications Ltd. California. U.S.A. 87 p.

Bovio, G. y A. Camia. 1997: Land Zoning Based on Fire History. Int. J. Wildland Fire. Vol. 7, No. 3. U.S.A. 249 - 258 pp.
Cuadras, C. M. 1991: Métodos de análisis multivariante. PPU. Barcelona. España. Limpergraf, S.A. 644 p.

González, A. 1997: Problemas de salinización en el acuífero litoral del occidente de Huelva. Servicio de publicaciones de la Universidad de Huelva. 284 - 288 pp.

MINAG. 1982: Incendios Forestales. Términos y definiciones. Norma Ramal de la Agricultura 555. Cuba. 7 p.

Ramos, M. P. 2002: Sistema Integrado para el Manejo de Bases de Datos sobre Incendios Forestales (SIMBDIF) Versión 1.2. Trabajo presentado en el III Congreso Forestal Venezolano.

Soares, R. V. 1985: Incêndios Florestais. Controle e uso do Fogo. Fundação de Pesquisas Florestais do Paraná. Curitiba, 213 p.

Soares, R.V. 1992: Ocurrência de incêndios florestais em reflorestamentos. I Seminario Nacional sobre Incêndios Florestais e Queimadas. Brasil, 15 p. 